别是 HPV 阳性患者 ) 的治疗具有积极的意义。

原文链接

http://onlinelibrary.wiley.com/doi/10.1002/cncr.29100/abstract

\title{
类风湿因子增高的个体易患深静脉 血栓
}

晋川

类风湿因子 $(\mathrm{RF})$ 是类风湿性关节炎 $(\mathrm{RA})$ 诊断中最为常用的标记物，约 70\% 的 RA 患 者显示 RF 增高。已有研究表明，RA 患者的 $\mathrm{RF}$ 与炎症指标等呈正相关，因此 RF 还可以 作为 $\mathrm{RA}$ 疾病活动度评价标志物。

大规模流行病学调查结果表明，RA 患者 发生静脉血栓的风险要高于健康个体，其原因 在于 RA 患者体内持续的炎症反应。尽管炎症 反应在静脉血栓发生与发展中的具体机制尚不 明确，但已有确丵的证据表明炎症指标增高的 个体患血栓性疾病的风险增高。

已知在普通人群中，约有 5\%-15\% 的个体 $\mathrm{RF}$ 增高。那么，这些 RF 增高但并未患 $\mathrm{RA}$ 的 个体发生血栓的风险是否也较高呢? 2015 年 2 月发表在 Clinical Chemistry 上的一项来自丹 麦的研究为我们揭示了答案。

该研究是一项大规模、基于普通人群的前 瞻性队列研究, 总共纳人了来自两个队列研究

(哥本哈根城心脏研究和哥本哈根普通人群研 究）的 54628 名普通人群, 并对这些人进行了 最长达 32 年的随访，用以分析基线 $\mathrm{RF}$ 水平 与静脉血栓风险是否相关。

在随访期间内，共有 670 名个体发生了 深静脉血栓（DVT），539名个体发生了肺栓 塞 ( PE )。通过统计学分析后研究者发现, $\mathrm{RF}$ 大于 $100 \mathrm{IU} / \mathrm{ml}$ 的个体，1 年和 5 年内发生

DVT 的风险分别是 RF 小于 $100 \mathrm{IU} / \mathrm{ml}$ 的个体 的 9 倍和 4.3 倍。进一步按照基线 RF 水平将 患者分为 5 组后, 研究者发现随着 RF 水平的 增高，患者发生 DVT 的风险具有逐渐增高的 趋势。

该研究同时也指出, $\mathrm{RF}$ 水平增高与 $\mathrm{PE}$ 的 发生风险无关。此外，在亚组分析中，研究 者还发现对于体重指数（BMI）大于 $30 \mathrm{~kg} / \mathrm{m} 2$ 且年龄在 60 岁以上的人群中，若 RF 高于 120 $\mathrm{IU} / \mathrm{ml}$ ，则男性和女性发生 DVT 的风险分别 为 $10 \%$ 和 $8 \%$ ；与之对应的是，若 $\mathrm{RF}$ 低于 15 $\mathrm{IU} / \mathrm{ml}$ ，男性和女性发生 DVT 的风险则仅为 $2.2 \%$ 和 $1.6 \%$ 。

该研究结果提示我们，对于 RF 增高的个 体，即使其未患 RA，也应该警惕 DVT 的发 生，尤其是对于年龄在 60 周岁以上、BMI 在 $30 \mathrm{~kg} / \mathrm{m} 2$ 以上且 $\mathrm{RF}$ 在 $120 \mathrm{IU} / \mathrm{ml}$ 以上的个体。

\section{原文链接}

http://www.clinchem.org/content/61/2/349.long

肿瘤病学

Oncology

\section{新型重组减毒单核李斯特菌技术治 疗恶性肿瘤}

Dr. Jiu Jiang

随着肿瘤学、免疫学以及分子生物学等相 关学科的快速发展和交叉渗透，肿瘤免疫治疗 的研究迅速发展。肿瘤疫苗的基本原理是利用 肿瘤抗原激发机体自身的免疫保护机制，达到 治疗肿瘤或预防复发的作用。

既往研究表明，基因修饰改造后的减毒细 菌能够在肿瘤的治疗中直接破坏肿瘤细胞或者 传递杀伤肿瘤细胞分子。而由于单核李斯特菌 (Listeria monocytogenes，LM) 能在专职吞 噬细胞和非专职吞噬细胞内生存繁殖, 引起强 烈的细胞免疫应答，所以单核李斯特菌在肿瘤 
免疫治疗的应用逐渐受到人们的重视。

将李斯特杆菌的两个致病毒力基因（分别 为 delta ActA 和 delta inlB ) 去除后，该菌可以 作为基因载体制备临床应用的减毒重组 $L M$ 。 而 LM 是目前用于肿瘤治疗中最为新颖且具有 广泛应用前景的肿瘤疫苗载体。将外源性肿瘤 特异性抗原基因导人减毒 LM 中, 就可以显著 诱导机体抗肿瘤细胞的免疫应答。

近年来, 位于美国加州伯克莱的 Aduro 公 司研制出表达间皮素 (mesothelin) 的减毒 LM 菌株，这种间皮素可在多种恶性肿瘤中高效表 达。感染表达间皮素的重组 LM 可刺激机体产 生有效的针对间皮素的杀伤 T 细胞 ( CTL) 免 疫反应, 从而清除肿瘤细胞及组织, 达到治疗 肿瘤的目的。

目前, 该重组 LM 已于 2014 年 7 月获美 国 FDA 突破性疗法（Breakthrough Therapy） 批准, 正在对胰腺癌开展二期临床试验。最近 发布的初步临床试验结果显示, 胰腺癌转移患 者在使用表达间皮素的重组 LM 后，总体生存 时间（OS）显著延长。此外, OS 的延长与间 皮素特异性 CD8 T 细胞应答增强呈正相关。

重组 LM 技术治疗肿瘤有以下几个特点:

(1) 经过不同的临床试验，已充分证明 应用不含 delta ActA 和 delta inlB 的减毒 LM,
具有较高的安全可靠性。

（2）重组 LM 培养条件要求不高, 易于 获取大量可用于临床治疗的工程菌。

(3) 与病毒载体不同, 重组 LM 不被中 和抗体抑制，因此可以重复使用。

（4）在治疗肿瘤时, 重组 LM 具有特异 性高、功效强的特点。诱发产生的 CTL 可同 时杀伤肿瘤原发灶及多发性转移病灶, 而对正 常组织无作用。

(5) 重组 LM 刺激机体 CTL 杀伤并清除 肿瘤组织后，可产生针对肿瘤抗原的免疫记忆 CD8 T 细胞, 可防止肿瘤的复发。

( 6 )既可单独使用, 也可与其他传统方法, 如化疗和放疗等联合使用。

\section{原文链接}

1.Le DT, Wang-Gillam A, Picozzi V, et al. Safety and Survival With GVAX Pancreas Prime and Listeria Monocytogenes-Expressing Mesothelin (CRS-207) Boost Vaccines for Metastatic Pancreatic Cancer. J Clin Oncol. 2015 Jan 12. pii: JCO.2014.57.4244. [Epub ahead of print]

2.Lizotte PH, Baird JR, Stevens CA, et al. Attenuated Listeria monocytogenes reprograms M2-polarized tumor-associated macrophages in ovarian cancer leading to iNOS-mediated tumor cell lysis. Oncoimmunology. 2014 May 23;3:e28926. eCollection.

3.Le DT, Brockstedt DG, Nir-Paz R, et al. A live-attenuated Listeria vaccine (ANZ-100) and a live-attenuated Listeria vaccine expressing mesothelin (CRS-207) for advanced cancers: phase I studies of safety and immune induction. Clin Cancer Res. 2012;18(3):858-68.

4.Sinha G. Listeria vaccines join the checkpoint frenzy. Nat Biotechnol. 2014;32(12):1176-7.

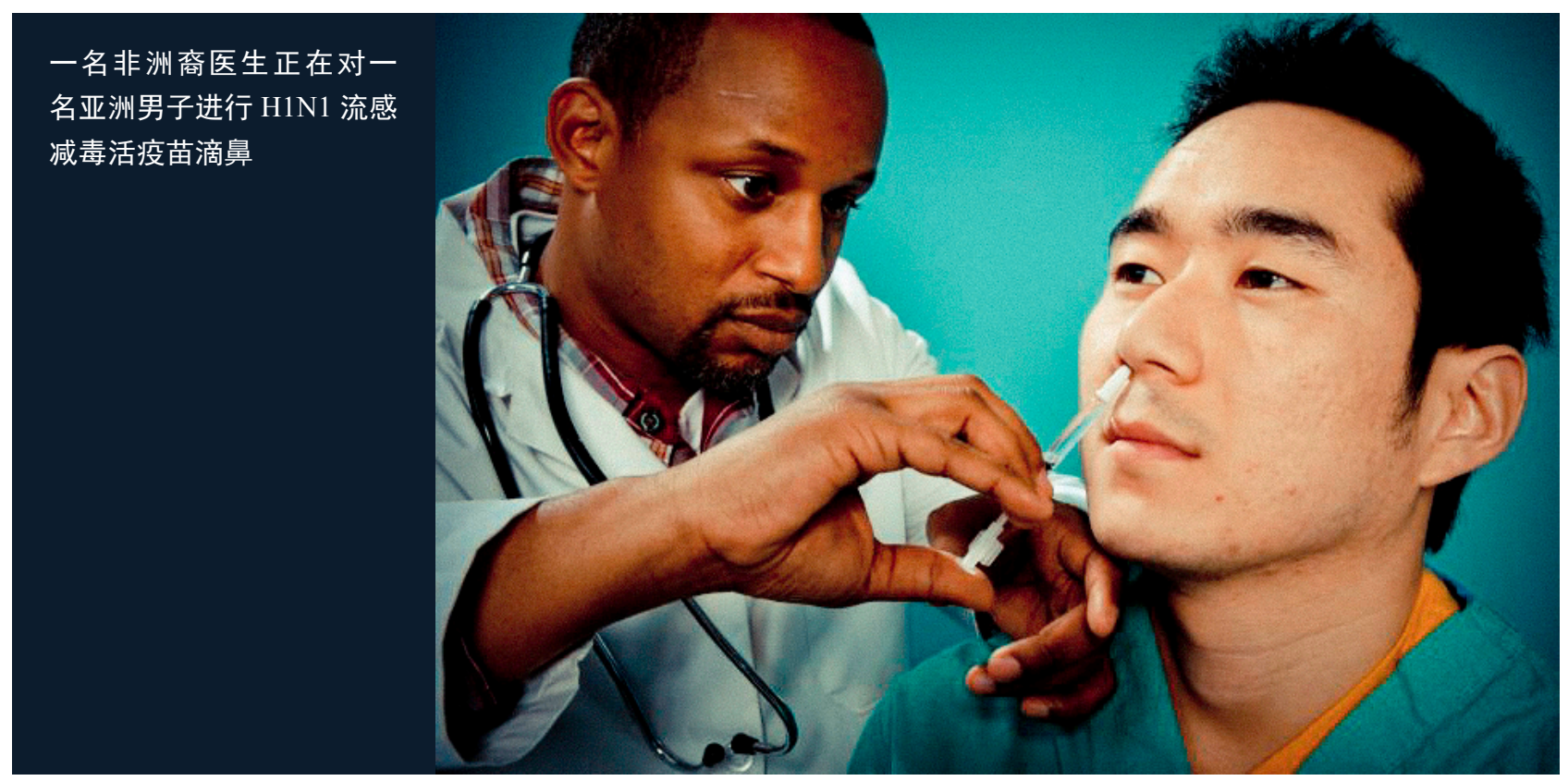

